\title{
Structure of the HeI $4^{3} \mathrm{D},{ }^{3} \mathrm{~F}-2{ }^{3} \mathrm{P}(\lambda=447.15 \mathrm{~nm}, \lambda=447.0 \mathrm{~nm})$ Spectral Line Emitted from a Low-Pressure Linear Discharge
}

\author{
H. W. Drawin and J. Ramette \\ Association EURATOM-CEA sur la Fusion \\ Département de Physique du Plasma et de la Fusion Contrôlée, Centre d'Etudes Nucléaires, \\ 92260 Fontenay-aux-Roses (France)
}

Z. Naturforsch. 33a, 1285-1293 (1978); received July 18, 1978

The line profile of the HeI line at $\lambda=447.15 \mathrm{~nm}$ has been measured during the ionisation phase and during the afterglow of a condensed low-pressure helium discharge by means of a 10-channel spectrum analyzer having a channel width of $0.1 \AA$. The temporal resolution was $1.6 \mu \mathrm{s}$ (e-folding time). The filling pressure ranged from one to twenty Torr. The same relative peak structure was observed during the plasma build-up phase and the afterglow, only the intensities of the peaks were different in the two cases. Our measured profiles are quite similar to those obtained under different experimental conditions by other authors who explained the structure as being due to Baranger-Mozer plasma satellites. In contrast to this we did not find any correlation between electron density and peak position, i.e. the interpretation as BarangerMozer plasma satellites has to be excluded. The wavelengths of a number of peaks agree well with already identified $\mathrm{He}_{2}$ molecular lines, and there are sufficient experimental indications to conclude that the prominent part of the satellite structure originates from $\mathrm{He}_{2}$ molecular transitions most of which have not yet been identified.

\section{Introduction}

Several authors have found characteristic structures in the profile of the $\mathrm{HeI}$ line $4^{3} \mathrm{D},{ }^{3} \mathrm{~F}-2^{3} \mathrm{P}$ when it was emitted by a plasma during turbulent heating or the first beginning of a discharge [1-7], during laser excitation [8] and plasma beam-gas target interaction [9], and during the afterglow $[6,10,11]$. Similar structures have also been observed for other HeI lines, e.g. [2, 12-15]. The features were explained as being due to plasma turbulence at the electron plasma frequency $\omega_{\text {pe }}$ which produces Baranger-Mozer plasma satellites [16] at wavelength distances $\pm \Delta \lambda_{\text {pe }}, \pm 3 \Delta \lambda_{\text {pe }}, \ldots$ from the forbidden component $4^{3} \mathrm{~F}-2^{3} \mathrm{P}$ and at wavelength distances $\pm 2 \Delta \lambda_{\text {pe }}, \pm 4 \Delta \lambda_{\text {pe }}, \ldots$ from the allowed transition $4^{3} \mathrm{D}-2^{3} \mathrm{P}$.

During our earlier measurements [17] of this HeI line we found during the late afterglow similar structures which were irregular in intensity from one shot to the other and which strongly depended on the general discharge conditions. Therefore these irregularities were smoothed out by the large number of measurements which led to the final profiles published in [17]. As we were at the time essentially interested in Stark-broadened profiles we did not pay much attention to the satellite structure.

Reprint requests to Mons. H.W. Drawin, Association Euratom-CEA, Département Fusion, Centre d'Etudes Nucléaires, Boite Postale $n^{\circ} 6$, F-92260 Fontenay-auxRoses, France.
We have now thoroughly repeated the measurements under very different discharge conditions and have analyzed the temporal evolution of the spectrum intensities during single discharges (shots) by means of a 10-channel spectrum analyzer system under refined conditions. We measured independently the temporal evolution of the electron density $N_{\mathrm{e}}$ with a He-Ne laser interferometer. We were thus able to determine $\omega_{\text {pe }}$, and consequently $\Delta \lambda_{\text {pe }}$, as a function of time independent of other spectroscopic measurements. If electron plasma satellites occur with sufficient intensity they should be detectable in a known spectral region within the profile during a well-defined time interval; in other words, the change of their wavelength position should be observable. There were no indications that Baranger-Mozer plasma satellites exist under our experimental conditions. In the contrary, we found coincidence of peak positions with $\mathrm{He}_{2}$ molecular transitions. From our measurements we must conclude that most of the peaks superposed on the HeI line profile belong to not yet identified $\mathrm{He}_{2}$ molecular transitions.

\section{Experimental}

\subsection{Discharge Tube, Electrical Circuit}

The experimental set-up was identical to the one described in $[17,18]$, only minor modifications were made. The plasma was produced in a linear discharge tube the construction of which is shown in 
Fig. 1 of [18]. For the present experiments we worked with an effective plasma length of $0.50 \mathrm{~m}$ instead of $0.20 \mathrm{~m}$. The inner diameter was $0.025 \mathrm{~m}$. The tube was filled with spectroscopically pure helium gas which was replaced after every five to ten shots, in some cases every two to three shots.

For the measurements of $N_{\mathrm{e}}$ the time constant was $1 \mu \mathrm{s}$ (e-folding time of electrical circuit plus response time of laser interferometer), the 10 channel analyzer system had a time constant of $1.6 \mu \mathrm{s}$. The time constant of the discharge circuit was dictated by these values in order to ensure that the plasma did not change its properties faster than we could measure them with sufficient precision and high reproducibility. For the measurements during the ionisation phase the discharge circuit therefore contained an inductance $(L=250 \mu \mathrm{H})$ which ensured the appropriate increase of the plasma current and, thus of $N_{\mathrm{e}}$. The afterglow measurements were performed after short-circuiting of the plasma current by an ignitron directly connected to the electrodes of the discharge tube. For further technical details, see [19].

\subsection{Performance of the 10-Channel Analyzer}

The 10-channel analyzer system was of same construction as the one described in [17]. The measuring head - attached to a Bausch and Lomb two meter dual-grating spectrograph in EbertFastié mounting - consisted of ten parallel, optically isolated rows of glass fibers. Every channel covered a spectral range of $0.1 \AA$, for a given position of the measuring head in the focal plane the spectral range covered was $1 \AA(+15 \%,-0 \%)$.

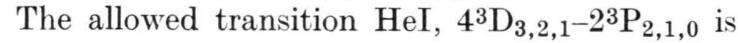
composed of a triplet of which two components corresponding to the transitions $4^{3} \mathrm{D}_{3}-2^{3} \mathrm{P}_{2}$ and $4^{3} \mathrm{D}_{2}-2^{3} \mathrm{P}_{1}$ fall practically together, thus only two components appear with a wave-length separation of approximately $0.21 \AA$. Calculations of Barnard et al. [20] show that the doublet character of the allowed component should become visible for $N_{\mathrm{e}} \leqq 2 \cdot 10^{13} \mathrm{~cm}^{-3}$ and $T \leqq 5000^{\circ} \mathrm{K}$ (see Table $3 \mathrm{a}$ in [20]). When correctly calibrated and positioned the 10-channel system should thus resolve the doublet structure at the corresponding values of $N_{\mathrm{e}}$ and $T_{\mathrm{e}}$. Figure 1 shows the temporal evolution of $N_{\mathrm{e}}$ for a set of measurements, Fig. 2 shows the evolution of the allowed transition $4^{3} \mathrm{D}-2^{3} \mathrm{P}$ during the late afterglow when the temperature is lower than $2500^{\circ} \mathrm{K}$ [17]. The doublet structure begins to emerge at $t \approx 140 \mu$ s after short-circuiting of the plasma current and is fully developed for times $t>160 \mu \mathrm{s}$. From the extrapolated curve in Fig. 1 follows $N_{\mathrm{e}}<2 \cdot 10^{13} \mathrm{~cm}^{-3}$ for $t>150 \mu \mathrm{s}$. The appearance of the doublet thus agrees with the theoretical predictions. The measured intensity ratio of the two components agrees within the experimental uncertainty with the theoretical ratio of $8 / 9: 1 / 9$.

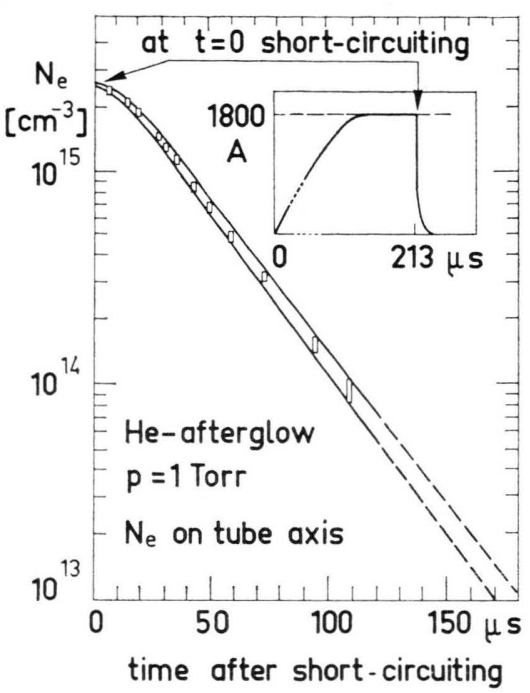

Fig. 1. Example for an electron density measurement in the region of the discharge axis during the afterglow. Filling pressure $p=1$ Torr, plasma current before shortcircuiting $1800 \mathrm{~A}$ (see insertion).

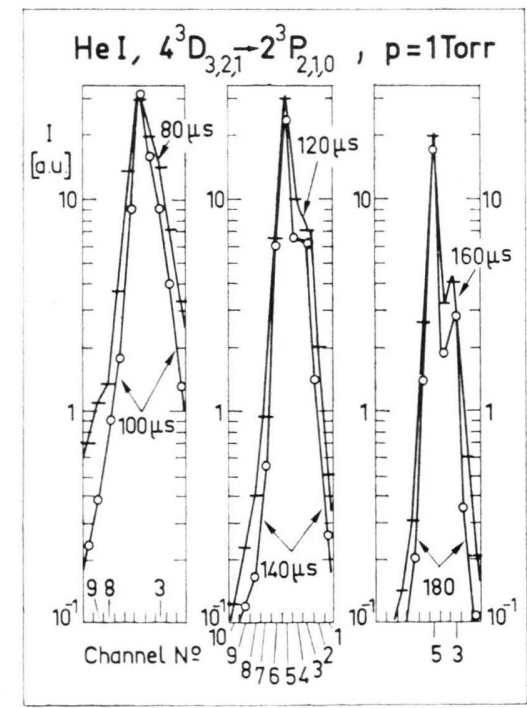

Fig. 2. Temporal evolution of the profile of the allowed line for plasma conditions given in Figure 1. 


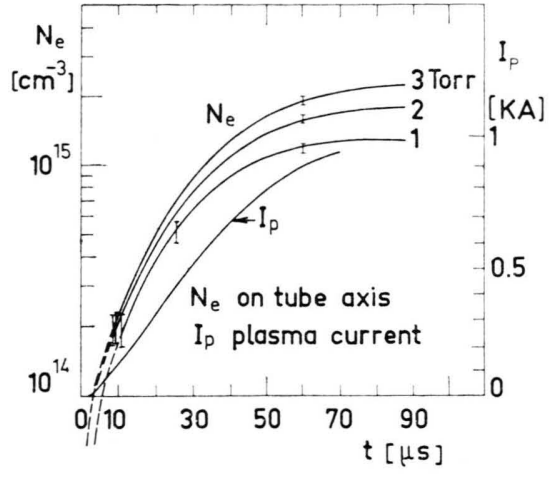

Fig. 3. Temporal evolution of plasma current $I_{\mathrm{p}}$ and electron density $N_{\mathrm{e}}$ on tube axis during ionisetion phase.

We have also resolved the fine structure during the very first beginning of the ionisation phase whose evolution of plasma current and electron density are shown in Figure 3. It follows from this, that the analyzer system measures correctly the temporal evolution of the line profiles with a spectral solution of $0.1 \AA$.

For further details the reader is refered to [19]. It should finally be mentioned that the maximum error between the 10-channels does not exceed $\pm 7.5 \%$ for a single shot.

\section{Results}

In order to obtain the entire profile from individual shots one has to displace the measuring head. This has been done in different steps in order to have overlapping of spectral bands. Measurements were made in the axial region of the discharge tube and in a region close to the wall. All spectroscopic measurements were made parallel to the tube axis in order to avoid Abel inversion. Self-absorption was insignificant except for high pressures [19].

According to the time bases chosen on the oscilloscopes, profiles were drawn in steps of one, two, five or ten microseconds. The few profiles shown in this article represent the essence of 252 shots with 2520 evaluated photographs for the ionisation phase (see Fig. 3) and of more than 500 shots with more than 5000 evaluated photographs for the afterglow plasmas. This large number of evaluated photographs has been chosen in order to eliminate as much as possible statistical errors in the intensity measurements and, thus, to avoid a false interpretation of the measurements. Due to very large number of measurements the very small error bars have not especially been indicated in the figures. For further details see [19].

\subsection{Ionisation Phase}

Typical profiles are shown in Figs. 4 and 5 emitted from the central region of the cylindrical plasma column. Quite similar profiles with still more pronounced peak structures and the same peak positions were obtained when observing the plasma near the wall. At $t=0$, the ignitron received the ignition pulse. Light emission began two to three microseconds later; the photomultiplier signals were well measurable for times $t \geqq 4 \mu$ s. Since the absolute intensities within a channel do not change strongly with time, the profiles in Figs. 4 and 5 have been displaced relative to each other parallel to the ordinate, thus avoiding overlapping and crossing of the curves.

The essential features are: During the build-up of the plasma, the doublet structure of the allowed line disappears and at later times the maximum of both the allowed and the forbidden component are shifted in opposite directions due to increasing intermolecular Stark effect. The profiles are strongly hashed due to the presence of many individual "peaks". Compared to the central intensity of the

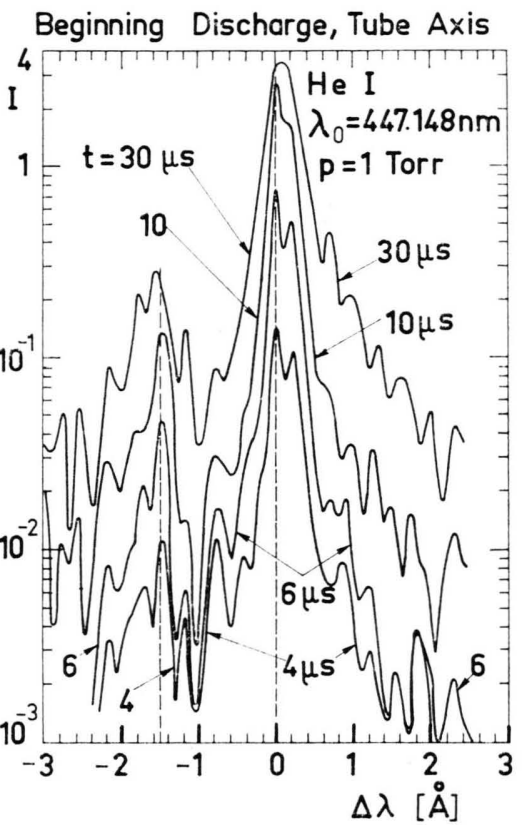

Fig. 4. Temporal evolution of the HeI line at the beginning of the discharge. Filling pressure $p=1$ Torr, intensity in arbitrary units. Emission from tube axis. Curves pertaining to different times are displaced along the I-direction. 


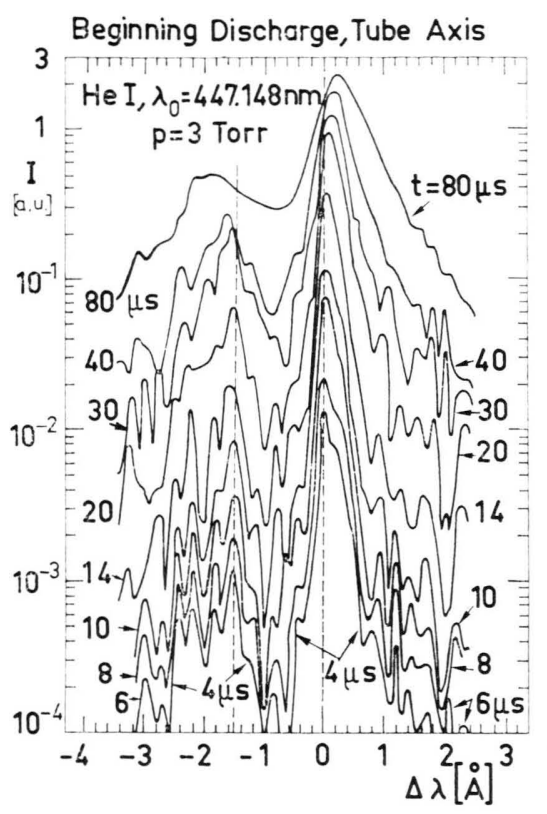

Fig. 5. Line profiles at different times during the ionisation phase. Filling pressure $p=3$ Torr. Intensity in arbitrary units. Emission from tube axis.

allowed line, these peaks have practically negligible intensity, but in the wings of the Stark-broadened line their contributions can be dominant. At same times $t$, the peak structure is more pronounced for higher filling pressures. At early times, both the allowed and forbidden line are narrow and the individual peaks are well distinguishable from each other. When time evolves the peak structure slowly disappears. For $t>100 \mu \mathrm{s}$ (not shown) one measures a "well-behaved" Stark-broadened line.

During the first twenty to thirty microseconds, the peak structure as a whole changes only little, although the intensities of some peaks relative to each other vary strongly.

Despite the large number of measurements no correlation between $N_{\mathrm{e}}$ and the evolution of the peak positions could be found.

As mentioned in the introduction, several authors have interpreted the peaks emerging from the generally Stark-broadened line wings as plasma satellites. The most recent measurements are those of Kawasaki [5] and Sanchez and Bengtson [6] who also had the highest spectral resolution compared to earlier publications.

In Kawasaki's experiment, the line was emitted from a small Theta-pinch device filled with $20 \mathrm{mTorr}$ pure helium gas. His published profile (the only one and taken at $t=0.7 \mu \mathrm{s}$ ) is reproduced in Fig. 6 together with the measuring points and error bars. The position of the forbidden component is at $\mathrm{F}$, the near and for Baranger-Mozer plasma satellites are typified by $S_{N}$ and $S_{F}$.

Sanchez and Bengtson produced their plasma in a linear discharge tube $(0.93 \mathrm{~m}$ long and $0.45 \mathrm{~m}$ in diameter). The filling pressure was $20 \mathrm{mT}$ Torr but it contained only $18 \%$ helium in hydrogen. Their published profile (only one taken at $t=40 \mu \mathrm{s}$ ) is also reproduced in Figure 6. F is the forbidden line, $\mathrm{S}_{-}$and $\mathrm{S}_{+}$denote the near and far Baranger-Mozer satellites with respect to $\mathrm{F}$, and $\mathrm{S}_{2-}$ and $\mathrm{S}_{2+}$ are the near and far plasma satellites with respect to the allowed line.

In both papers the profile was obtained on a shot-to-shot basis with a single photomultiplier. In order to obtain a profile, the grating had to be tilted.

Both profiles are compared with our measurements. To this end we have added in Fig. 6 one of our profiles measured at early time of the discharge. The lowest curve in Fig. 6 represents the profile for $t=4 \mu$ s of Fig. 4 , but also a profile at later times could have been taken.

Despite the very different discharge conditions in the three experiments, the observed peak structure is similar. Especially the positions of the prominent peaks agree within $\pm 0.05 \AA$, that is within a precision given by the channel width of $0.1 \AA$ in our experiment. The slightly less structured

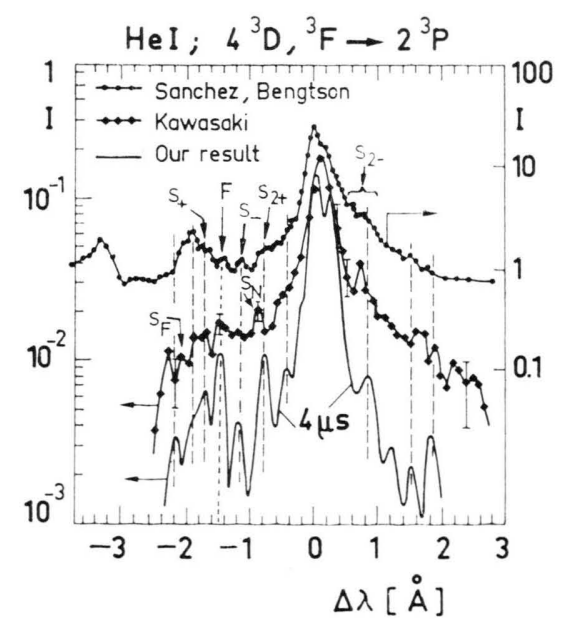

Fig. 6. Comparison of the profiles measured by Kawasaki [5] $\left(N_{\mathrm{e}}=1 \cdot 10^{14} \mathrm{~cm}^{-3}\right)$, Sanchez and Bengtson [6] $\left(N_{\mathrm{e}} \approx 1.6 \cdot 10^{14} \mathrm{~cm}^{-3}\right)$ with our profile at $t=4 \mu \mathrm{s}$ $\left(N_{\mathrm{e}} \approx 1 \cdot 10^{13}\right.$ to $\left.2 \cdot 10^{13} \mathrm{~cm}^{-3}\right)$ during the ionisation phase. 
profile of Sanchez and Bengtson can easily explained by the lower helium concentration (see conclusion).

Similar agreement between our peak structures and those measured by other authors is obtained when one takes into account the relatively bad spectral resolution in earlier experiments.

There is also a great similarity between the profiles of Sanchez and Bengtson [6] and Pistunovich et al. [9]. In the latter paper, the broad intensity bump with its maximum situated at $\Delta \lambda \approx-3.3 \AA$ and interpreted as plasma satellite $\mathrm{S}_{+}$would lead to $N_{\mathrm{e}} \approx 8.5 \cdot 10^{14} \mathrm{~cm}^{-3}$. This high value is only obtained when one adds to the plasmoid density $N_{\mathrm{e}^{\prime}} \approx 2.5 \cdot 10^{14} \mathrm{~cm}^{-3}$ the helium gas target density $N_{0} \approx 4 \cdot 10^{14} \mathrm{~cm}^{-3}$, with the additional assumption that complete ionisation was achieved and that one still allows for an uncertainty of $50 \%$ in the density measurements. However, for a practically similar structure and density distribution over wavelength in [6] the measured electron density in only $N_{\mathrm{e}} \cong 1.6 \cdot 10^{14} \mathrm{~cm}^{-3}$. This suggests that there might be a contradiction in the interpretation of the measured features.

\subsection{Afterglow}

Figure 7 resumes the results obtained when the line is emitted from the axial region at a filling pressure of $p=1$ Torr. During the very early time of the afterglow (short-circuiting is at $t=0 \mu \mathrm{s}$ ) the line profiles are "well-behaved" and need not be shown. They revealed practically no special structure (except at rather high filling pressures) apart from the existence of the electron densityshifted forbidden line. Sometimes appearing slight irregularities in the profile can be assumed to be due to a "bad shot". Eliminating such a shot or averaging over a larger number of shots with a "bad shot not eliminated" yields practically the same result, since the irregularities smooth out when one takes the mean values. One thus obtains quasistructureless Stark-broadened profiles which are relatively well described by the theoretical calculations of Griem [21] and Barnard et al. [22]. This is in agreement with our earlier measurements [17] of this line under somewhat other discharge conditions.

As the time evolves, the profiles become narrower and peaks emerge first in the far line wings. Towards the end of the afterglow, the peaks can well be distinguished very close to the line center. Several peaks occur between the allowed and forbidden lines. The intensity of the peaks relative to the intensity of the HeI line increases with increasing filling pressure (measurements made for $p=2,3$ and 10 Torr are not shown in the present paper). The same phenomenon was observed during the ionisation phase of the plasma (see Figs. 4 and 5).

As for the ionisation phase, the intensity of the peaks changes with time without any significant change of wavelength position. The wavelengths of most of the peaks at the end of the afterglow agree with the peak positions observed at the first beginning of the discharge, however, the peak intensities are very different in the two cases. In the following, some of the observed peculiarities shall be discussed.

In Fig. 7 which refers to a filling pressure of 1 Torr, the most interesting feature appears between allowed and forbidden transitions. At early times after short-circuiting the valley has no special structure. Between $30 \mu$ s and $40 \mu s$ a weak but broad dip occurs which becomes more and pronounced with time. At $60 \mu$ s this dip splits up in two components. The intensity of one component decreases during the later afterglow and a deeps intensity minimum appears, whereas the intensity of the other component remains relatively strong without changing wavelength. Some finer features in the profile do not appear on the figure, since the wavelength and intensity scales are too compressed. At the long-wavelength side the allowed transition has a broad shoulder around a wavelength distance of 1 to $1.5 \AA$ showing a characteristic peak structure

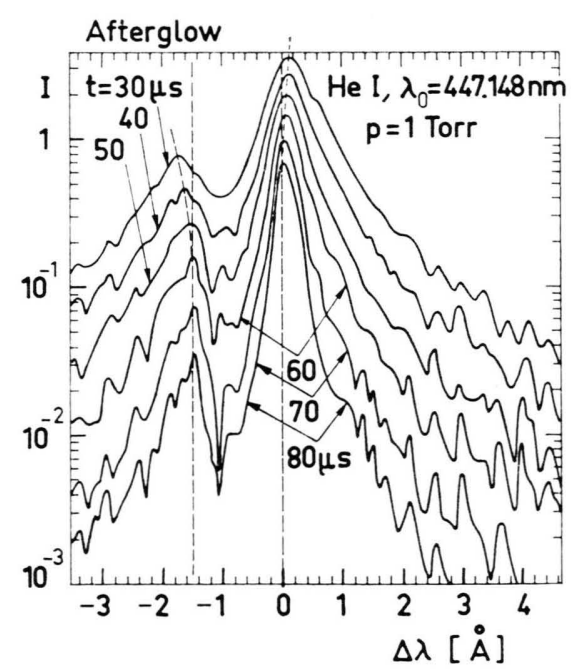

Fig. 7. Line profiles emitted from afterglow plasma in axial region. Filling pressure $p=1$ Torr. 
quite similar to the one observed during the ionisation phase.

Profiles measured in the wall region are shown in Figs. 8 and 9. In Fig. 9, our result refers to a time $t=90 \mu \mathrm{s}$ after short-circuiting of the plasma current.

Without a few exceptions (see [19]) no correlation between peak position and $N_{\mathrm{e}}$ could be found.

Recently Sanchez and Bengtson [6] also studied the $\mathrm{HeI}$ line at $\lambda=447.15 \mathrm{~nm}$ when it was emitted from an afterglow plasma of low electron density

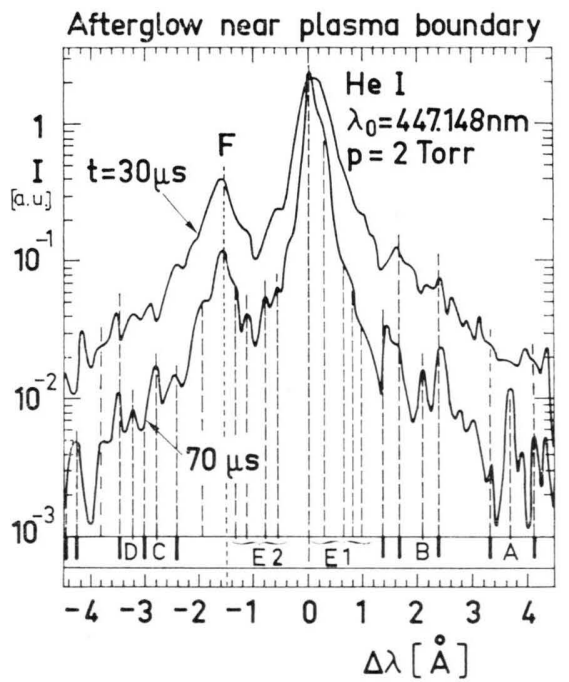

Fig. 8. Line profiles measured during afterglow near the wall. Fat lines just above abscisse represent identified $\mathrm{He}_{2}$ molecular lines after [23].

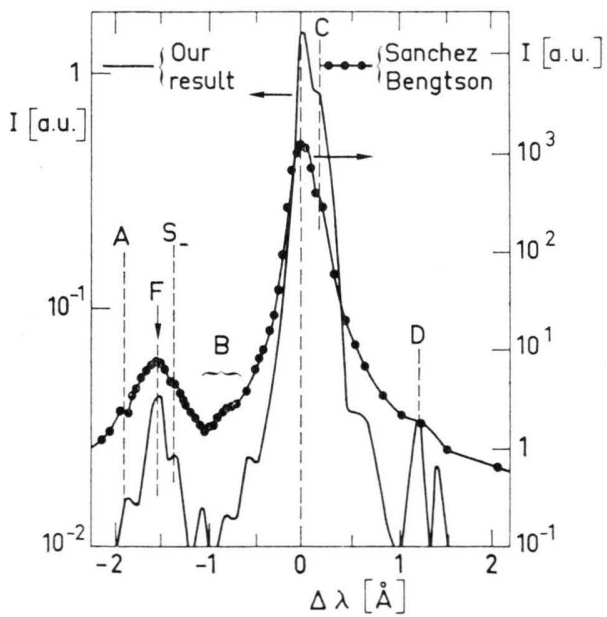

Fig. 9. Comparison of Sanchez-Bengtson profile [6] with our profile observed at $t=90 \mu \mathrm{s}$ during the afterglow and emitted from the wall region. A, B, C, D are irregularities not discussed in [6].
$\left(N_{\mathrm{e}}=10^{14} \ldots 10^{15} \mathrm{~cm}^{-3}\right)$. In their experiment the filling gas contained $18 \%$ helium in hydrogen. In the publication no indications are given about the current level during the afterglow phase. Their published line profile is shown in Fig. 9 and compared with our profile obtained during the late afterglow $(t=90 \mu \mathrm{s}$, near the wall). Sanchez and Bengtson observed slight irregularities of which one was interpreted as the near plasma satellite $\mathrm{S}_{-}$. The far plasma satellite is obviously missing.

Our measured profile emitted from a pure helium plasma refers to a much lower electron density and shows a peak whose position agrees with the $\mathrm{S}_{-}$peak. All other irregularities in the SanchezBengtson profile appear at wavelengths where we resolve peaks which have $N_{\mathrm{e}}$-independent positions.

\section{Conclusion and Interpretation}

The line profiles measured during the ionisation phase and the afterglow showed many individual peaks the wavelengths of which could not be related to $N_{\mathrm{e}}$. Comparison of our profiles obtained during the plasma build-up phase with those of other authors [5-6] measured during turbulent heating of helium plasmas had shown that the peak structure is qualitatively identical. When one compares our profiles obtained during the afterglow with the profile of Sanchez and Bengtson [6] also obtained during the afterglow one finds the same similarity although not so pronounced as in the first case. These facts and the observation that the peak intensities increase with increasing pressure and that their wavelength positions are practically independent of $N_{\mathrm{e}}$ have led us to the conclusion that the satellite structure - interpreted by other authors as Baranger-Mozer plasma satellites - does in the majority of cases not originate from plasma oscillations.

There are sufficient indications to conclude that the prominent part of the satellite structure originates from $\mathrm{He}_{2}$ molecular lines belonging to the electronic bands $n^{\prime}=4 \rightarrow n^{\prime \prime}=2$. Figure 10 shows the positions of $\mathrm{He}_{2}$ lines in the region of the $\mathrm{HeI} 4^{3} \mathrm{D},{ }^{3} \mathrm{~F}-2^{3} \mathrm{P}$ transition, calculated from the values published by Brown and Ginter [23]. The figure contains also a schematic drawing of the HeI line profile. Only rotational lines belonging to vibrational transitions $v^{\prime}=0 \rightarrow v^{\prime \prime}=0, v^{\prime}=1 \rightarrow$ $v^{\prime \prime}=1$ and $v^{\prime}=2 \rightarrow v^{\prime \prime}=2$ have yet been identified 


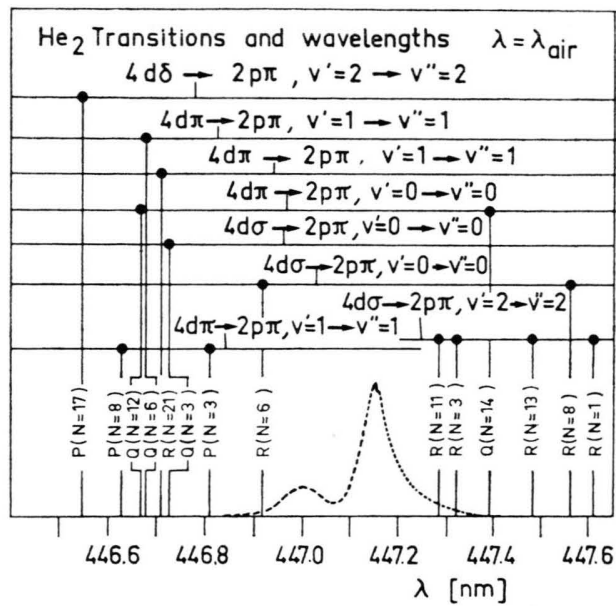

Fig. 10. The known wavelengths of $\mathrm{He}_{2}$ transitions in the region of the $\mathrm{HeI} 4^{3} \mathrm{D},{ }^{3} \mathrm{~F}-2^{3} \mathrm{P}$ transition calculated from the tables in [23].

with the exception of those lines which fall in the wavelength region of the HeI transition. When one draws the Fortrat parabola for the R-branch of the $4 d \sigma j^{3} \Sigma_{\mathrm{u}}{ }^{+}\left(v^{\prime}=2\right) \rightarrow 2 p \pi b^{3} \Pi_{\mathrm{g}}\left(v^{\prime \prime}=2\right)$ transitions one sees that the band head nearly coincides with the allowed transition $4^{3} \mathrm{D}-2^{3} \mathrm{P}$ and that the missing lines $\mathrm{R}(N=5), \mathrm{R}(N=7), \mathrm{R}(N=9)$ must be superposed on the near wings between allowed and forbidden lines. This is the reason why they could not yet be identified in photographic measurements which integrate light during the whole discharge.

In Fig. 8 we have compared the measured peak structure with the wavelengths of identified molecular transitions. The short fat lines just above the abscissa represent the known rotational lines shown in Figure 10. Within the spectral resolving power of our device, known molecular lines correspond to peaks in the measured line profiles.

There are several strong peaks denoted by A, B, $\mathrm{C}, \mathrm{D}, \mathrm{E}_{1}, \mathrm{E}_{2}$ which are preferentially emitted during the very first beginning of the ionisation phase or during the late afterglow, others are visible during the whole afterglow phase. It is possible that they represent also molecular transitions, but between higher vibrational quantum numbers whose transitions have not yet been investigated. However, three of the peaks of the group $\mathrm{E}_{2}$ should represent the above mentioned three missing lines of the R-branch of

$$
4 d \sigma j^{3} \Sigma_{\mathrm{u}}+\left(v^{\prime}=2\right) \rightarrow 2 p \pi b^{3} \Pi_{\mathrm{g}}\left(v^{\prime \prime}=2\right)
$$

for which we find the following most probable wavelengths:

$$
\begin{array}{ll}
\mathrm{R}(N=5): & \lambda_{\text {air }}=447.10 \pm 0.01 \mathrm{~nm}, \\
\mathrm{R}(N=7): & \lambda_{\text {air }}=447.04 \pm 0.04 \mathrm{~nm}, \\
\mathrm{R}(N=9): & \lambda_{\text {air }}=447.07 \pm 0.01 \mathrm{~nm} .
\end{array}
$$

One of the peaks at the wavelengths

$$
\lambda_{\text {air }}=446.93 \mathrm{~nm} ; 4469.80 \mathrm{~nm} ; 4470.20 \mathrm{~nm}
$$

probably represents the $\mathrm{He}_{2}$ line

$$
4 d \pi j^{3} \Sigma_{\mathrm{u}}\left(v^{\prime}=1\right) \rightarrow 2 p \pi b^{3} \sum_{\mathrm{g}}\left(v^{\prime \prime}=1\right),
$$

$\mathrm{Q}(N=8)$ of the Q-branch.

The interpretation of the peak structure as being due to molecular lines is in agreement with most of the still other observations:

- the peak intensities vary strongly with discharge conditions without changing position;

- the peak intensities increase with increasing filling pressure,

- the peak structure becomes more and more complexe when the filling pressure is increased, since more of otherwise weak molecular lines will emerge from the background intensity, see [19] were further examples are given.

Also the relatively weak peaks in the SanchezBengtson profiles (Figs. 6 and 9) find a natural explanation: In addition to $\mathrm{He}_{2}$ molecular lines there appear molecular lines of $\mathrm{H}_{2}$ and possibly of $\mathrm{HHe}$ which partially mask the $\mathrm{He}_{2}$ lines. Indeed, the strong intensity bump in Fig. 6 with its maximum at $\Delta \lambda=-3.3 \AA$ corresponds to the most intense $\mathrm{H}_{2}$ lines in this region [24] and many other closely spaced lines of weaker intensity are situated in this wavelength range. In the light of these results measurements of the satellite structure of the $\mathrm{H}_{\beta}$ line of atomic hydrogen [18] have to be revisited. Profiles obtained from new measurements over a broad range of plasma parameters will be published soon.

When this interpretation of the prominent part of the peak structure is correct, the following conclusions must be drawn: first, structures observed in the helium lines and interpreted by various authors as plasma satellites were probably $\mathrm{He}_{2}$ molecular lines; second, spectral and time resolved intensity measurements by means of a multi-channel spectrum analyzer would offer a 
unique method to identify molecular transitions from transient plasmas even in regions of intense Stark-broadened lines, since the intensity in the line wings changes with time and will be low when molecular emission is intense (low degree of ionisation). In a transient state the thermodynamic properties of a plasma change continuously, i.e. also the relative populations of excited states change continuously. A measurement of the temporal evolution of the intensity of molecular lines could thus be a considerable help in the identification of lines originating from states with different upper quantum numbers.

Burgess [25] has published a line profile of the C-IV transitions $5{ }^{2} \mathrm{G},{ }^{2} \mathrm{~F},{ }^{2} \mathrm{D}-4^{2} \mathrm{~F},{ }^{2} \mathrm{D}$ on which two satellites are superposed. These latter have been interpreted as Baranger-Mozer plasma satellites. Until now this is the only case where such satellites have been observed in the spectrum of ionised atoms. However, quite another explanation might be possible. Due to the strong non-thermal excitation in the investigated Theta-pinch plasma autoionisation states of $\mathrm{C}^{2+}$ may be excited. Thus, the C-IV lines of $\mathrm{C}^{3+}$ can be accompanied by lines originating from transitions between autoionising states of $\mathrm{C}^{2+}$. Due to the high intensities of the observed satellites this possibility seems to be more likely than the formation of Baranger-Mozer plasma satellites. Only a detailed study of the energy levels of the autoionising states of $\mathrm{C}^{2+}$ can decide whether this interpretation is correct or not.

It should be mentioned that not all of our observations fit completely in the picture of $\mathrm{He}_{2}$ molecular transitions superposed on the wings of the $\mathrm{HeI}$ line. In some rare cases we could identify weak intensity bumps whose wavelength positions are probably related to $N_{\mathrm{e}}$. Also strong shifts of both the allowed and forbidden HeI lines at low electron density and a sometimes occuring dip in the center of the allowed line remain unexplained. These special cases are presented and discussed in [19].

Further investigations of the "peak structure" of the helium lines will necessitate multi-channel spectrum analyzers of higher resolving power and covering a much larger spectral range. Also refined diagnostics (for instance fluorescence measurements with dye laser) seem to be necessary to relate the structure of the line profiles quantitatively to the plasma parameters*.

\section{Acknowledgements}

The technical assistance of Mr. E. Sablon during the measuring campaign and the exploitation of the great number of photographs is gratefully acknowledged.

* Note added in proof: Burgess et al. (Proceedings of contributed papers, Summer School and Symposium on the Physics of Ionized Gases, pp. 393-396, August 28September 2, 1978, Dubrovnik/Yugoslavia) reported on similar measurements of profiles of the $\mathrm{H}_{\alpha}$ and the HeI, $\lambda=5876 \AA\left(3^{3} \mathrm{D}-2^{3} \mathrm{P}\right)$ lines made during the afterglow of a ten meter long plasma. In the far line wings of $\mathrm{H}_{\alpha}$ appeared a structure "connected with hydrogen rather than with impurity species". - The published HeI line shows a structure which is different in the blue and red wings. A peak at $\Delta \lambda=0.30 \AA$ is due to fine structure splitting (the theoretical value is $0.345 \AA$ ). There seems to emerge a weak peak at $\Delta \lambda=0.65 \AA$, and a further peak is clearly resolved at $\Delta \lambda=2.45 \AA$. On the blue side a plateau appears in the region between $\Delta \lambda=-0.3 \AA$ and $\Delta \lambda=-1.0 \AA$, probably due to an unresolved structure. Finally a weak peak is visible at $\Delta \lambda=-3.0 \AA$.

The features at $\Delta \lambda=2.45 \AA$ and $\Delta \lambda=-3.0 \AA$ have been interpreted by the authors as Baranger-Mozer plasma satellites for the measured electron density of $3 \cdot 10^{14} \mathrm{~cm}^{-3}$ (measured "with very high precision").

In our opinion, these measurements confirm our interpretation of molecular lines, due to the following facts. First, for $N_{\mathrm{e}}=3 \cdot 10^{14} \mathrm{~cm}^{-3}$ the satellites should occur at wavelength distances $\Delta \lambda= \pm 1.87 \AA$ from the forbidden line $3^{3} \mathrm{P}-2^{3} \mathrm{P}$ at $6069 \AA$ or at $\Delta \lambda= \pm 3.5 \AA$ from the allowed line $3^{3} \mathrm{D}-2^{3} \mathrm{P}$ at $5876 \AA$. This is not observed. Second, the perturbing levels $3^{3} \mathrm{P}_{0,1,2}$ have a mean energy distance of $537 \mathrm{~cm}^{-1}$ from the $3^{3} \mathrm{D}$ levels (for comparison, the difference between the $4^{3} \mathrm{~F}$ and $4^{3} \mathrm{D}$ levels is only $7.8 \mathrm{~cm}^{-1}$ ). Thus, the satellite intensities are by several orders of magnitude weaker than those to the $4^{3} \mathrm{~F}-2^{3} \mathrm{P}$ transition and become too faint to be observable. It is very likely that the observed features have the same origin as in our experiment, namely transitions between different electronic states of the $\mathrm{H}_{2}$ and $\mathrm{He}_{2}$ molecule. Inspection of the potential energy curves of $\mathrm{He}_{2}$ shows that the electronic states $n=3, f^{3} \Sigma_{\mathrm{u}}{ }^{+}, f^{3} \Pi_{\mathrm{u}}, f^{3} \Delta_{\mathrm{u}}$ and $n=2, b^{3} \Pi_{\mathrm{g}}$ are involved.
[1] H.-J. Kunze and H. R. Griem, Phys. Rev. Letters 21, 1048 (1968).

[2] Y. Hamada, J. Phys. Soc. Japan 29, 463 (1970).

[3] F. R. Scott, R. V. Neidigh, J. R. McNally jr., and W. S. Cooper, J. Appl. Physics 41, 5327 (1970).

[4] W. R. Rutgers, Z. Naturforsch. 30a, 1271 (1975).
[5] K. Kawasaki, J. Phys. Soc. Japan 43, 648 (1977).

[6] A. Sanchez and R. D. Bengtson, Phys. Rev. Letters 38, 1276 (1977).

[7] E. Möbius, Thesis, Ruhr-Universität, Bochum (F.R.G.) 1977. 
[8] C. F. Burrel and H.-J. Kunze, Phys. Rev. Letters 29 , 1445 (1972).

[9] V. I. Pistunovich, V. V. Platonov, V. D. Ryutov, and E. A. Filimonova, Soviet Phys. J.E.T.P. Letters 23, 26 (1976).

[10] G. Baravian, R. Benatar, J. Bretagne, J. Godart, and G. Sultan, Physics Letters 30a, 198 (1969).

[11] G. Baravian, J. Bretagne, J. Godart, and G. Sultan, Z. Physik B 20, 247 (1975), B 20, 255 (1975).

[12] H.-J. Kunze, H. R. Griem, A. W. De Silva, G. C. Goldenbaum, and I. J. Spalding, Physics Fluids 12, 2669 (1969).

[13] N. Ben-Yosef and A. G. Rubin, J. Quant. Spectrosc. Radiat. Transfer 11, 1 (1971).

[14] W. D. Davis, Physics Fluids 15, 2383 (1972).

[15] C. C. Gallagher and M. A. Levine, J. Quant. Spectrosc. Radiat. Transfer 15, 275 (1975).

[16] M. Baranger and R. Mozer, Phys. Rev. 123, 25 (1961).
[17] H. W. Drawin and J. Ramette, Z. Naturforsch. 29a, 838 (1974).

[18] J. Ramette and H. W. Drawin, Z. Naturforsch. 31 a, 401 (1976).

[19] H. W. Drawin and J. Ramette, Report EUR-CEAFC 963, Fontenay-aux-Roses, July 1978.

[20] A. J. Barnard, J. Cooper, and E. W. Smith, J. Quant. Spectrosc. Radiat. Transfer 14, 1025 (1974).

[21] H. R. Griem, Astrophys. J. 154, 1111 (1968).

[22] A. J. Barnard, J. Cooper, and L. J. Shamey, Astron. Astrophys. 1, 28 (1969).

[23] C. M. Brown and M. L. Ginter, J. Mol. Spectrosc. 46, 256 (1973).

[24] H. M. Crosswhite, The Hydrogen Molecular Wavelength Tables of Gerhard Heinrich Dieke, Wiley Intersci., New York 1972.

[25] D. D. Burgess, Space Sci. Rev. 13, 493 (1972). 